\title{
Explaining COVID-19 Vaccine Rejection Using Social Cognitive Theory in Qassim, Saudi Arabia
}

\author{
Aseel Ali AlSaeed *(D) and Unaib Rabbani (D) \\ Family Medicine Academy, Qassim Health Cluster, Buraidah 52385, Saudi Arabia; rabbaniunaib@gmail.com \\ * Correspondence: aseel_alsaeed@hotmail.com; Tel.: +966-502923873
}

Citation: AlSaeed, A.A.; Rabbani, U. Explaining COVID-19 Vaccine Rejection Using Social Cognitive Theory in Qassim, Saudi Arabia. Vaccines 2021, 9, 1304. https:// doi.org/10.3390/vaccines9111304

Academic Editors: Tiziana Ramaci and Massimiliano Barattucci

Received: 16 October 2021

Accepted: 8 November 2021

Published: 9 November 2021

Publisher's Note: MDPI stays neutral with regard to jurisdictional claims in published maps and institutional affiliations.

Copyright: (c) 2021 by the authors. Licensee MDPI, Basel, Switzerland. This article is an open access article distributed under the terms and conditions of the Creative Commons Attribution (CC BY) license (https:/ / creativecommons.org/licenses/by/ $4.0 /)$.

\begin{abstract}
Acceptance of COVID-19 vaccines needs a health promotion approach to address various social, environmental and personal factors leading to vaccine hesitancy. We assessed the vaccine hesitancy rate and applied social cognitive theory (SCT) to understand COVID-19 vaccine rejection in Qassim, Saudi Arabia. A cross-sectional study was conducted among visitors of 10 randomly selected primary health care centers in Buraidah, Saudi Arabia. Data was collected by a self-administrated questionnaire. The variables were grouped into six constructs of SCT. Logistic regression was used to assess the predictors of vaccine rejection. Out of 486 participants included in the study, $30.5 \%$ rejected the vaccine. The most common reason for vaccine rejection was uncertainty about the vaccine's effectiveness (78\%). Among various constructs of SCT, reciprocal determinism (nationality, income and suffering from COVID-19 infection), behavioral capability (knowledge about vaccine safety), self-efficacy (registered for vaccine), and observational learning (getting the vaccine after friends and family members) were significant predictors. Expectation and reinforcement constructs did not show significant association. There was high vaccine rejection in Qassim, KSA. This calls for further improving the mass education strategies. Social cognitive theory can be used to predict vaccine rejection and to develop strategies to increase the utilization of COVID-19 vaccines in Saudi Arabia.
\end{abstract}

Keywords: COVID-19; vaccine hesitancy; barriers to vaccine; social cognitive theory; Saudi Arabia

\section{Introduction}

Millions of people worldwide have been infected and/or died due to severe acute respiratory syndrome coronavirus 2 (SARS-CoV-2) or COVID-19 infection since the start of the pandemic [1]. Referring to the number of cases and deaths, this pandemic is a serious threat to global public health systems [2].

Developing an effective and safe vaccine was a promising hope since the early days of the pandemic. Vaccination is considered one of the most effective strategies to control the pandemic along with other measures such as social distancing, masks, and the use of sanitizers [3]. However, the availability of a vaccine is not enough to control the pandemic. Vaccine acceptance is very important to assess, as it reflects the overall perception of disease risk in the population [4]. A number of factors associated with rejection have been reported in various studies such as vaccine safety and efficacy, lack of trust in health system, misconception, and misinformation. All these factors can lead to low vaccination rates and jeopardize public health $[5,6]$.

Globally, vaccine acceptance has been widely studied and has shown wide variations. A global survey that included participants from 19 countries reported that about $75 \%$ of the respondent were "very to somewhat" likely to accept COVID-19 vaccination [7]. A systematic review that included studies from 33 countries reported acceptance rates to be highest (more than 90\%) in Ecuador, Malaysia, Indonesia, and China. On the other hand, Kuwait (23.6\%) and Jordan (28.4\%) showed the lowest acceptance rates [8]. Two different surveys that included participants from more than 19 Arab countries reported vaccine acceptance to be between $62 \%$ and $58.5 \%[9,10]$. Similarly, a number of studies 
have been published from Saudi Arabia, which reported vaccine acceptance rates to range from $48 \%$ to $72 \%$ [5,6,11-13]. The common barriers reported among the Saudi population include concerns about the safety and efficacy of vaccines, vaccines being a conspiracy, and a perceived low risk of COVID-19 infection $[6,11,12]$. Studies have also reported various factors associated with vaccine uptake which include fear of infection, level of trust of vaccines, level of trust in the health care system, age, gender, marital status, education, presence of chronic diseases, and previous influenza vaccine uptake $[4,10,12,13]$.

Vaccine acceptance is very important to assess, as the success of any vaccination programs depend on the public's willingness to take it. There are many theories from social sciences that have been used to understand health behaviors and promotion of healthy behaviors. Some of these theories are used at an individual level while others are used at group and community levels. Vaccine acceptance, while a personal decision, is influenced by various environmental and social factors. Previous studies have used these theories to explain the vaccine acceptance rate among individuals and groups. Studies on influenza, human papilloma virus, and hepatitis B vaccinations have used such theories to explain the factors associated with acceptance of these vaccines [14-16]. Recently, a number of studies have used these theories to understand COVID-19 vaccination behaviors. Most of these studies have mainly used the health belief model (HBM) [17-21] and only few have used social cognitive theory (SCT) [22]. Nonetheless, these studies have been able to predict the COVID-19 vaccine utilization rate at an individual level. However, COVID-19 vaccination is also affected by various factors outside of an individual such as behaviors of others towards vaccination, governmental policies regarding vaccination, and media exposure.

The Saudi government took various steps to curb the spread of COVID-19, which included lockdowns, travel restrictions, use of masks and hand sanitizers, and mass education about prevention of disease. Furthermore, in the month of April 2021, the government made it mandatory to be vaccinated to visit the Holy sites of Makkah and Madinah. Later, the General authority for civil aviation (GACA) announced that starting from 17 May, travelling outside the Kingdom will only be allowed for vaccinated persons. Despite all these measures widespread vaccine rejection has been reported.

Vaccination is critical for controlling the current COVID-19 pandemic. Resistance towards vaccination is a threat to global public health. It is therefore necessary to address this issue through a health promotion approach. SCT, an interpersonal framework, can be helpful in explaining the personal and social factors influencing vaccine rejection and help develop strategies to improve the utilization of COVID-19 vaccines in the general population. This study therefore aimed to assess COVID-19 vaccine acceptance and apply SCT to explain the rejection of vaccines and its barriers among primary health care (PHC) center attendees in the Qassim region of Saudi Arabia.

\section{Materials and Methods}

\subsection{Study Design and Setting}

A cross-sectional study was conducted among the attendees of selected primary health care centers in Buraidah from February 2021 to June 2021. Buraidah is the capital of Qassim region with an estimated population of 693,515 [23].

\subsection{Sample Size}

The sample size for the study was calculated using WHO software for sample size determination in health studies. The sample size was measured using $44.7 \%$ prevalence of COVID-19 vaccination acceptance [6]. At 95\% confidence level and 5\% bound on error, the maximum calculated sample size was 380 participants.

\subsection{Sampling Technique and Procedure}

There are 40 primary health care centers in Buraidah. Out of these, 10 centers were selected by a simple random sampling. Within each selected PHC, a total of 40-50 participants were selected consecutively by convenience sampling. Males and females $\geq 18$ years 
of age and citizens and residents were eligible to participate. Those who had vaccine contraindication according to Saudi Ministry of Health $(\mathrm{MoH})$ (having a history of a severe allergic reaction, pregnant women, those planning to conceive in next 2 months, and those within 90 days of COVID-19 infection at the time of data collection) were excluded from the study. The purpose of the study and its objectives were explained to the patients who were in the waiting rooms. Participants were assessed for eligibility. Those meeting eligibility criteria were invited to participate in the study.

\subsection{Data Collection Tool}

Data was collected by a structured questionnaire in Arabic. The questionnaire consisted of five domains. The domains aimed to collect data on socio-demographic variables, awareness about COVID-19 and its vaccine, vaccine acceptance, reasons for not opting for COVID-19 vaccination, and sources of COVID-19 vaccine information. The questionnaire was developed after a review of the literature [3-6,24]. Study variables were identified to meet the objectives of the study. The draft questionnaire was reviewed by research faculty in the program and finalized.

The items were organized into six constructs of SCT. Socio-demographic characteristics and history of COVID-19 infection in family or individual were grouped in reciprocal determinism. Knowledge and practices related to COVID-19 infection prevention were kept under behavioral capabilities. Risk and severity of infection were included in expectations. Intention and registration to receive COVID-19 vaccine constituted self-efficacy. Observational learning was assessed by the question "I will get COVID-19 vaccine only if my friends or other family members get it first" while reinforcement construct included a vaccine requirement by an employer and enforcement by the government.

The questionnaire was translated into Arabic first and then back-translated to English by an independent translator for validation of the Arabic translation of the questionnaire. A pilot study was conducted to ensure the clarity of questions, applicability to the participants, and to identify if there was any problem that could have impeded the data collection process. After pilot testing, the questionnaire was modified according to the observations during testing.

\subsection{Data Collection Procedure}

Data was collected by trained data collectors who were either doctors or nurses. First, the study purpose and procedure were explained and informed consent was obtained. Printed questionnaires were provided to the participants. Participants were required to fill out the form by themselves; data collectors were available to assist if any clarification was required.

\subsection{Data Entry and Analysis}

Data was entered and analyzed using SPSS version 23. Descriptive analysis was carried out in the form of frequencies and percentage for categorical variables while mean and standard deviation (SD) were calculated for continuous variables. Logistic regression analysis was used to assess the predictors of vaccine rejection. Variables that had a $p$-values of $\leq 0.2$ in the univariate analysis were included in the multivariate models. Variables in the final model were retained based on their significance and effects on -2 log likelihood ratio. Adjusted and unadjusted odds ratio along with associated $95 \%$ confidence interval were calculated. A $p$-value $<0.05$ was considered significant for all inferential analysis.

Ethical approval of the study was taken from the Qassim Regional Bioethics Committee. Informed consent was obtained from all participants.

\section{Results}

A total of 486 participants completed the survey questionnaire of which $54 \%$ were males. Fifty-four percent were married and a majority (90.5\%) were Saudi nationals. Approximately one third (34.8\%) had a high school or lower educational level, whereas 
$65.2 \%$ had a diploma or higher. Regarding health status, $84.2 \%$ did not have any chronic disease and $28.7 \%$ had received an influenza vaccine during the previous year. Around $16 \%$ reported having suffered from COVID-19 while $37.3 \%$ had one of their family members infected with the disease (Table 1).

Table 1. Socio-demographic and health characteristics of the study population $(n=486)$.

\begin{tabular}{|c|c|c|}
\hline \multicolumn{2}{|l|}{ Variable } & \multirow{2}{*}{$\frac{\%(n)}{32.80(12.08)}$} \\
\hline Age $(n=474)$ & Mean (SD) & \\
\hline \multirow[t]{2}{*}{ Gender } & Male & $54(262)$ \\
\hline & Female & $46(223)$ \\
\hline \multirow[t]{2}{*}{ Marital status } & Never married & $46.1(219)$ \\
\hline & Ever Married & $53.9(256)$ \\
\hline \multirow[t]{2}{*}{ Nationality } & Saudi & $90.5(428)$ \\
\hline & Non-Saudi & $9.5(45)$ \\
\hline \multirow[t]{2}{*}{ Educational level } & High school and lower & $34.8(165)$ \\
\hline & Diploma and higher & $65.2(309)$ \\
\hline \multirow[t]{2}{*}{ Employment status } & Unemployed & $53.6(254)$ \\
\hline & Employee & $46.4(220)$ \\
\hline \multirow[t]{4}{*}{ Monthly Income } & Less than 5000 SAR & $46.6(194)$ \\
\hline & $5001-10,000$ SAR & $26(108)$ \\
\hline & 10,001-20,000 SAR & $23.6(98)$ \\
\hline & More than 20,000 SAR & $3.8(16)$ \\
\hline \multirow[t]{2}{*}{ Any chronic disease } & No & $84.2(405)$ \\
\hline & Yes & $15.8(76)$ \\
\hline \multirow[t]{2}{*}{ Did you get influenza vaccine in last two years? } & No & $71.3(338)$ \\
\hline & Yes & $28.7(136)$ \\
\hline \multirow[t]{2}{*}{ Did you suffer from COVID-19? } & No & $84.1(397)$ \\
\hline & Yes & $15.9(75)$ \\
\hline \multirow[t]{2}{*}{$\begin{array}{l}\text { Did any of your family members suffer from } \\
\text { COVID-19? }\end{array}$} & No & $62.7(296)$ \\
\hline & Yes & $37.3(176)$ \\
\hline \multirow[t]{3}{*}{$\begin{array}{l}\text { In your opinion your risk of getting COVID-19 } \\
\text { infection is }\end{array}$} & Less than $10 \%$ & $33.3(148)$ \\
\hline & $10-40 \%$ & $39.8(177)$ \\
\hline & $>40 \%$ & $27(120)$ \\
\hline
\end{tabular}

Table 2 shows participants' awareness and behaviors towards COVID-19. It was found that $68.2 \%$ believed COVID-19 could lead to death and 91.9\% reported that COVID19 spreads by close contact with infected people. A majority $(97.7 \%)$ were aware of the COVID-19 vaccine and $61 \%$ believed it to be safe. Half (50.9\%) of the respondents had already registered for COVID-19 vaccination and $28 \%$ were waiting for their family or friends to be vaccinated before they would be vaccinated. 
Table 2. Awareness and behaviors towards COVID-19 and its vaccines.

\begin{tabular}{|c|c|}
\hline Variable & $\%(n)$ \\
\hline \multicolumn{2}{|c|}{ COVID-19 disease could lead to death } \\
\hline Yes & $68.2(324)$ \\
\hline No & $11.4(54)$ \\
\hline Not sure & $20.4(97)$ \\
\hline \multicolumn{2}{|c|}{ COVID-19 may lead to hospitalization } \\
\hline Yes & $87.8(417)$ \\
\hline No & $4.6(22)$ \\
\hline Not sure & $7.6(36)$ \\
\hline \multicolumn{2}{|c|}{ COVID-19 spreads by close contact to infected people } \\
\hline Yes & $91.9(434)$ \\
\hline No & $4(19)$ \\
\hline Not sure & $4(19)$ \\
\hline \multicolumn{2}{|c|}{ COVID-19 can be prevented by precautionary measures } \\
\hline Yes & $84.3(397)$ \\
\hline No & $6.4(30)$ \\
\hline Not sure & $9.3(44)$ \\
\hline \multicolumn{2}{|c|}{ I have heard about COVID-19 vaccine } \\
\hline Yes & $97.7(460)$ \\
\hline No & $1.7(8)$ \\
\hline Not sure & $0.6(3)$ \\
\hline \multicolumn{2}{|c|}{ COVID-19 vaccine is available in the Kingdom } \\
\hline Yes & $93.8(442)$ \\
\hline No & $1.9(9)$ \\
\hline Not sure & $4.2(20)$ \\
\hline \multicolumn{2}{|c|}{ COVID-19 vaccine is effective in preventing the disease } \\
\hline Yes & $63.2(299)$ \\
\hline No & $6.3(30)$ \\
\hline Not sure & $30.4(144)$ \\
\hline \multicolumn{2}{|c|}{ COVID-19 vaccine is safe } \\
\hline Yes & $61(288)$ \\
\hline No & $6.6(31)$ \\
\hline Not sure & $32.4(153)$ \\
\hline \multicolumn{2}{|c|}{ If available, I will get COVID-19 vaccine for my family member } \\
\hline Yes & $76.2(342)$ \\
\hline No & $23.8(107)$ \\
\hline \multicolumn{2}{|c|}{ I already registered for get COVID-19 vaccine } \\
\hline Yes & $50.9(223)$ \\
\hline No & $49.1(215)$ \\
\hline
\end{tabular}


Table 2. Cont.

\begin{tabular}{cc}
\hline Variable & $\%(n)$ \\
\hline I will get my vaccine only if required by my employer & \\
\hline Yes & $40.7(174)$ \\
\hline No & $59.3(253)$ \\
\hline Yes & \\
\hline No & $28(120)$ \\
\hline members get it first & $72(308)$ \\
\hline Y wash my hands frequently & $86.2(405)$ \\
\hline No & $13.8(65)$ \\
\hline I use sanitizer frequently & $74.3(349)$ \\
\hline Yes & $25.7(121)$ \\
\hline No & $90.9(430)$ \\
\hline I always wear mask in public places & $9.1(43)$ \\
\hline
\end{tabular}

Regarding the sources of information for COVID-19, the most common was TV/radio $(67.9 \%)$ followed by social media $(67.70 \%)$. Other sources of information included the Ministry of Health $(64.40 \%)$, friends and family members $(54.30 \%)$, health care providers (53.50\%), and newspapers (26.50\%).

The vaccine rejection rate was found to be $30.50 \%$. A significant difference was found in the vaccine rejection rates between pre- and post-enforcement periods (59.3\% vs. $40.7 \%$; $p$ value $<0.001)$.

The most commonly reported reason behind vaccine rejection was the belief that vaccines may not be effective $(78.4 \%)$ and the least common reason was that vaccines are a conspiracy (31.6\%) (Figure 1).

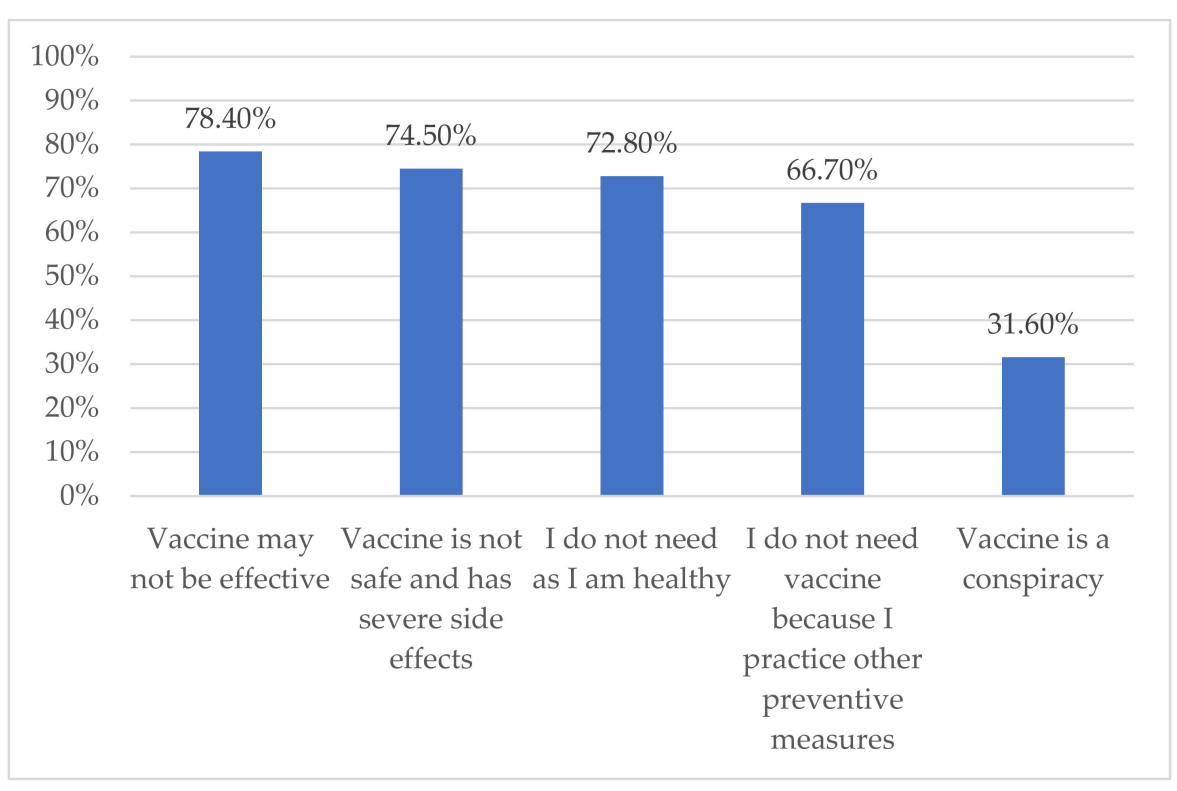

Figure 1. Reasons for rejecting COVID-19 vaccine. 
Multivariate logistic regression was performed to identify the influencing factors of vaccination rejection, which were grouped under various constructs of SCT. Significant predictors in the construct of reciprocal determinism included nationality (aOR $0.03,95 \% \mathrm{CI}$ 0.00-0.60), history of COVID-19 (aOR 3.29, 95\% CI: 1.08-9.98), an income of more than SAR 20,000 per month (aOR 10.13, 95\% CI: 15-89.17), and television as the main source of information about COVID-19 (OR 3.40, 95\% CI: 1.29-8.99). When considering behavior, being unsure about vaccine safety was associated with higher odds of vaccine rejection (aOR 4.92, 95\% CI: 1.85-13.11). None of the variables included in the expectancy construct were found to be significantly associated with vaccine rejection. In the self-efficacy category, those who did not register for vaccination were more likely to reject the vaccine. Observational learning was a significant predictor of vaccination, as those who did not follow friends and other family members were more than four times more likely to reject the vaccine (aOR 4.12, 95\% CI: 1.53-11.06). In the construct of reinforcement, there was no significant association of government enforcement with the decision of rejecting vaccines (Table 3).

Table 3. Association of vaccine rejection with various constructs of social cognitive theory in Qassim, Saudi Arabia.

\begin{tabular}{|c|c|c|c|c|}
\hline \multirow{2}{*}{ Variables } & \multicolumn{2}{|c|}{ Univariable } & \multicolumn{2}{|c|}{ Multivariable } \\
\hline & OR $(95 \% \mathrm{CI})$ & $p$-Value & OR $(95 \% \mathrm{CI})$ & $p$-Value \\
\hline \multicolumn{5}{|c|}{ Reciprocal determinism } \\
\hline \multicolumn{5}{|l|}{ Gender } \\
\hline Male & 1 & & 1 & \\
\hline Female & $1.30(0.88-1.92)$ & 0.188 & $1.72(0.75-3.93)$ & 0.199 \\
\hline \multicolumn{5}{|l|}{ Marital status } \\
\hline Never married & 1 & & & \\
\hline Ever Married & $1.13(0.76-1.68)$ & 0.550 & & \\
\hline \multicolumn{5}{|l|}{ Nationality } \\
\hline Saudi & 1 & & 1 & \\
\hline Non-Saudi & $0.27(0.10-0.69)$ & 0.007 & $0.03(0.00-0.60)$ & 0.022 \\
\hline \multicolumn{5}{|l|}{ Educational level } \\
\hline High school and lower & 1 & & & \\
\hline Diploma and higher & $1.12(0.74-1.70)$ & 0.599 & & \\
\hline \multicolumn{5}{|l|}{ Employment status } \\
\hline Unemployed & 1 & & & \\
\hline Employee & $0.95(0.64-1.41)$ & 0.799 & & \\
\hline \multicolumn{5}{|l|}{ Monthly Income } \\
\hline Less than SAR 5000 & 1 & & 1 & \\
\hline SAR 5001-10000 & $1.10(0.66-1.83)$ & 0.706 & $1.26(0.46-3.49)$ & 0.653 \\
\hline SAR 10001-20000 & $0.80(0.46-1.39)$ & 0.424 & $2.24(0.82-6.13)$ & 0.117 \\
\hline SAR More than 20000 & $1.02(0.34-3.06)$ & 0.976 & 10.13 (1.15-89.17) & 0.037 \\
\hline \multicolumn{5}{|l|}{ Any chronic disease? } \\
\hline No & 1 & & & \\
\hline Yes & $0.80(0.45-1.40)$ & 0.427 & & \\
\hline \multicolumn{5}{|l|}{ Did you get influenza } \\
\hline $\begin{array}{l}\text { vaccine in last two years? } \\
\text { No }\end{array}$ & 1 & & & \\
\hline Yes & $0.31(0.19-0.53)$ & $<0.001$ & & \\
\hline
\end{tabular}


Table 3. Cont.

\begin{tabular}{|c|c|c|c|c|}
\hline \multirow{2}{*}{ Variables } & \multicolumn{2}{|c|}{ Univariable } & \multicolumn{2}{|c|}{ Multivariable } \\
\hline & OR $(95 \%$ CI $)$ & $p$-Value & OR $(95 \%$ CI $)$ & $p$-Value \\
\hline $\begin{array}{l}\text { Did you suffer from } \\
\text { COVID-19? } \\
\text { No } \\
\text { Yes }\end{array}$ & $1.71(1.03-2.86)$ & 0.040 & $\begin{array}{c}1 \\
3.29(1.08-9.98)\end{array}$ & 0.036 \\
\hline $\begin{array}{l}\text { Did any of your family } \\
\text { members suffer from } \\
\text { COVID-19? } \\
\text { No } \\
\text { Yes }\end{array}$ & $\begin{array}{c}1 \\
1.28(0.86-1.92)\end{array}$ & 0.226 & $\begin{array}{c}1 \\
0.51(0.19-1.33)\end{array}$ & 0.168 \\
\hline $\begin{array}{l}\text { Television/radio } \\
\text { No } \\
\text { Yes }\end{array}$ & $\begin{array}{c}1 \\
1.87(1.24-2.81) \\
\end{array}$ & 0.003 & $\begin{array}{c}1 \\
3.40(1.29-8.99) \\
\end{array}$ & 0.013 \\
\hline $\begin{array}{l}\text { News papers } \\
\text { No } \\
\text { Yes }\end{array}$ & $\begin{array}{c}1 \\
2.20(1.35-3.59) \\
\end{array}$ & 0.002 & $\begin{array}{c}1 \\
1.22(0.39-3.81)\end{array}$ & 0.739 \\
\hline $\begin{array}{l}\text { Social Media (e.g., } \\
\text { Facebook, Twitter, } \\
\text { Instagram, WhatsApp) } \\
\text { No } \\
\text { Yes }\end{array}$ & $\begin{array}{c}1 \\
1.47(0.98-2.23)\end{array}$ & 0.065 & $\begin{array}{c}1 \\
1.52(0.42-5.47)\end{array}$ & 0.525 \\
\hline $\begin{array}{l}\text { Friends and Family } \\
\text { Members } \\
\text { No } \\
\text { Yes }\end{array}$ & $\begin{array}{c}1 \\
1.35(0.91-2.00)\end{array}$ & 0.134 & $1.33(0.40-4.35)$ & 0.641 \\
\hline $\begin{array}{l}\text { Ministry of Health's } \\
\text { COVID-19 related } \\
\text { information } \\
\text { Yes } \\
\text { No }\end{array}$ & $\begin{array}{c}1 \\
2.01(1.35-3.01)\end{array}$ & 0.001 & $0.57(0.16-2.00)$ & 0.377 \\
\hline $\begin{array}{l}\text { Health Care providers } \\
\text { Yes } \\
\text { No }\end{array}$ & $\begin{array}{c}1 \\
1.92(1.29-2.85) \\
\end{array}$ & 0.001 & $\begin{array}{c}1 \\
0.54(0.16-1.85)\end{array}$ & 0.328 \\
\hline & Behavioral & apability & & \\
\hline $\begin{array}{l}\text { COVID-19 spreads by close } \\
\text { contact to infected people } \\
\text { Yes } \\
\text { No } \\
\text { Not sure }\end{array}$ & $\begin{array}{c}1 \\
2.25(0.85-5.96) \\
4.34(1.67-11.27)\end{array}$ & $\begin{array}{l}0.104 \\
0.003\end{array}$ & $\begin{array}{c}1 \\
4.54(0.90-22.86) \\
2.36(0.28-20.15)\end{array}$ & $\begin{array}{l}0.066 \\
0.432\end{array}$ \\
\hline $\begin{array}{l}\text { COVID-19 can be prevented } \\
\text { by precautionary measures } \\
\text { Yes } \\
\text { No } \\
\text { Not sure }\end{array}$ & $\begin{array}{c}1 \\
1.59(0.73-3.48) \\
2.85(1.52-5.36)\end{array}$ & $\begin{array}{l}0.245 \\
0.001\end{array}$ & $\begin{array}{c}1 \\
0.36(0.06-2.02) \\
2.98(0.91-9.76)\end{array}$ & $\begin{array}{l}0.246 \\
0.071\end{array}$ \\
\hline $\begin{array}{l}\text { I have heard about } \\
\text { COVID-19 vaccine } \\
\text { Yes } \\
\text { No } \\
\text { Not sure }\end{array}$ & $\begin{array}{c}1 \\
14.09 \\
(1.68-118.15) \\
1.17(0.11-13.06)\end{array}$ & $\begin{array}{l}0.015 \\
0.896\end{array}$ & & \\
\hline
\end{tabular}


Table 3. Cont.

\begin{tabular}{|c|c|c|c|c|}
\hline \multirow{2}{*}{ Variables } & \multicolumn{2}{|c|}{ Univariable } & \multicolumn{2}{|c|}{ Multivariable } \\
\hline & OR $(95 \% \mathrm{CI})$ & $p$-Value & OR $(95 \%$ CI $)$ & $\begin{array}{c}p- \\
\text { Value }\end{array}$ \\
\hline $\begin{array}{l}\text { COVID-19 vaccine is } \\
\text { available in the Kingdom } \\
\text { Yes } \\
\text { No } \\
\text { Not sure }\end{array}$ & $\begin{array}{c}1 \\
7.44(1.48-37.36) \\
3.41(1.34-8.68)\end{array}$ & $\begin{array}{l}0.015 \\
0.010\end{array}$ & & \\
\hline $\begin{array}{l}\text { COVID-19 vaccine is } \\
\text { effective in preventing the } \\
\text { disease } \\
\text { Yes } \\
\text { No } \\
\text { Not sure }\end{array}$ & $\begin{array}{c}1 \\
21.30(8.21-55.22) \\
6.23(3.94-9.86)\end{array}$ & $\begin{array}{l}<0.001 \\
<0.001\end{array}$ & $\begin{array}{c}1 \\
4.44(0.80-24.62) \\
1.42(0.50-4.04)\end{array}$ & $\begin{array}{l}0.088 \\
0.507\end{array}$ \\
\hline $\begin{array}{l}\text { COVID-19 vaccine is safe } \\
\text { Yes } \\
\text { No } \\
\text { Not sure }\end{array}$ & $\begin{array}{c}1 \\
10.49(4.65-23.69) \\
7.53(4.73-11.97)\end{array}$ & $\begin{array}{l}<0.001 \\
<0.001\end{array}$ & $\begin{array}{c}1 \\
4.83(0.80-29.15) \\
4.924(1.85-13.11)\end{array}$ & $\begin{array}{l}0.086 \\
0.001\end{array}$ \\
\hline $\begin{array}{l}\text { I wash my hands } \\
\text { frequently } \\
\text { Yes } \\
\text { No }\end{array}$ & $4.34(2.50-7.54)$ & $<0.001$ & $2.84(0.89-9.10)$ & 0.078 \\
\hline $\begin{array}{l}\text { I use sanitizer frequently } \\
\text { Yes } \\
\text { No }\end{array}$ & $\begin{array}{c}1 \\
2.65(1.71-4.11)\end{array}$ & $<0.001$ & & \\
\hline $\begin{array}{l}\text { I always wear mask in } \\
\text { public places } \\
\text { Yes } \\
\text { No }\end{array}$ & $\begin{array}{c}1 \\
2.62(1.37-5.01)\end{array}$ & 0.004 & & \\
\hline \multicolumn{5}{|c|}{ Expectations } \\
\hline $\begin{array}{l}\text { In your opinion your risk } \\
\text { of getting COVID-19 } \\
\text { infection is } \\
\text { Less than } 10 \% \\
10-40 \% \\
>40 \%\end{array}$ & $\begin{array}{c}1 \\
0.92(0.58-1.47) \\
0.54(0.31-0.95)\end{array}$ & $\begin{array}{l}0.723 \\
0.031\end{array}$ & & \\
\hline $\begin{array}{l}\text { COVID-19 could lead } \\
\text { to death } \\
\text { Yes } \\
\text { No } \\
\text { Not sure }\end{array}$ & $\begin{array}{c}1 \\
1.90(1.03-3.53) \\
2.20(1.37-3.54)\end{array}$ & $\begin{array}{l}0.041 \\
0.001\end{array}$ & & \\
\hline $\begin{array}{l}\text { COVID-19 may lead to } \\
\text { hospitalization } \\
\text { Yes } \\
\text { No } \\
\text { Not sure }\end{array}$ & $\begin{array}{c}1 \\
2.26(0.90-5.71) \\
2.01(1.01-4.01)\end{array}$ & $\begin{array}{l}0.084 \\
0.048\end{array}$ & & \\
\hline \multicolumn{5}{|c|}{ Self-efficacy } \\
\hline $\begin{array}{l}\text { If available, I will get } \\
\text { COVID-19 vaccine for my } \\
\text { family member } \\
\text { Yes } \\
\text { No }\end{array}$ & $\begin{array}{c}1 \\
137.20(61.96-303.79)\end{array}$ & $<0.001$ & & \\
\hline
\end{tabular}


Table 3. Cont.

\begin{tabular}{|c|c|c|c|c|}
\hline \multirow{2}{*}{ Variables } & \multicolumn{2}{|c|}{ Univariable } & \multicolumn{2}{|c|}{ Multivariable } \\
\hline & OR $(95 \% \mathrm{CI})$ & $p$-Value & OR $(95 \% \mathrm{CI})$ & $p$-Value \\
\hline $\begin{array}{l}\text { I already registered for get } \\
\text { COVID-19 vaccine } \\
\text { Yes } \\
\text { No }\end{array}$ & $\begin{array}{c}1 \\
15.86(8.52-29.50)\end{array}$ & $<0.001$ & $\begin{array}{c}1 \\
20.80(6.99-61.87)\end{array}$ & $<0.001$ \\
\hline \multicolumn{5}{|c|}{ Observational learning } \\
\hline $\begin{array}{l}\text { I will get COVID-19 vaccine } \\
\text { only if my friends or other } \\
\text { family members get it first } \\
\text { Yes } \\
\text { No }\end{array}$ & $\begin{array}{c}1 \\
1.02(0.64-1.62)\end{array}$ & 0.947 & $\begin{array}{c}1 \\
4.12(1.53-11.06)\end{array}$ & 0.005 \\
\hline \multicolumn{5}{|c|}{ Reinforcements } \\
\hline $\begin{array}{l}\text { I will get my vaccine only if } \\
\text { required by my employer } \\
\text { Yes } \\
\text { No }\end{array}$ & $\begin{array}{c}1 \\
0.37(0.24-0.56)\end{array}$ & $<0.001$ & $\begin{array}{c}1 \\
0.20(0.09-0.46)\end{array}$ & $<0.001$ \\
\hline $\begin{array}{l}\text { Period } \\
\text { Pre enforcement } \\
\text { Post enforcement }\end{array}$ & $\begin{array}{c}1 \\
0.48(0.33-0.72)\end{array}$ & $<0.001$ & & \\
\hline
\end{tabular}

\section{Discussion}

In this study we estimated the COVID-19 vaccine rejection rate and barriers to vaccination and attempted to explain these by using social cognitive theory. We found that nearly one-third of respondents rejected the vaccines. The belief that vaccine may not be effective was the most common reason for rejection. Nationality, history of getting COVID-19, if friends or family received the vaccine, and reinforcement were significant predictors for the vaccine rejection.

We found that the rejection rate was $30.5 \%$, which is approximately consistent with the findings of another study done in Qassim. They collected data from March to May 2021 and reported that $14.7 \%$ refused vaccination and $22.7 \%$ were still undecided [25]. Rejection was higher in a study done by Magadmi et al. in Saudi Arabia, which reported a 55.3\% rejection rate [6]. Additionally, two studies were done in Saudi Arabia that reported the difference between vaccine rejection before and after the vaccine was available. Before the roll out of the vaccines only $7 \%$ refused the vaccine and $28.2 \%$ were not sure [5]. After the vaccine was available $46.7 \%$ of the participants reported that they would take it only if it is mandatory [13]. On the other hand, varying rates of vaccine rejection have been reported in Middle Eastern countries. The rejection rate was reported to be $45.2 \%$ in Qatar [26], while another study, which included participants from all the Arab countries, showed a $38 \%$ rejection rate [9]. Earlier studies on COVID-19 vaccination related behaviors from different countries showed low rejection rates: China, 8.7\% [18]; Israel, 20\% [19]; and Hong Kong 17\% [17]. Studies from low-income countries have generally reported higher rejection rates. For example, studies from Ghana and Ethiopia reported higher rejection rates of $46 \%$ and $37 \%$, respectively $[27,28]$. The variation in the vaccine hesitancy across the studies could be due to the differences in time when the studies were conducted, the setting of the study, and the local burden of COVID-19 infections. Our finding of a 30\% rejection rate should be considered as a high level of COVID-19 vaccine hesitancy, especially when it is freely available to all of the population and enforcement measures exist, such as making vaccination mandatory for entering holy places and shopping malls as well as for travelling outside the country. It is important to explore barriers behind hesitancy and rejection of COVID-19 vaccines in order to overcome these issues and reach the goal of an immunized society. Our study showed that the most common barriers were believing the vaccines are not effective, not safe, and have serious side effects. Similarly, a study done in the Qassim 
region showed the reason was mainly that people did not believe in the vaccines [25]. Other studies done in Saudi Arabia showed consistent results with our study. One study showed that concerns about the side effects were the barrier for most refusers $(80 \%)$, a lack of trust concerning the effectiveness of the vaccines was reported by $25 \%$, and only $15 \%$ believed in a conspiracy theory about vaccines [6]. Another study, showed that around half of participants refused the vaccine because of concerns about the effectiveness and for $33.1 \%$ of participants the reason for rejection was information from social media sources [11]. Alobaidi S, in his study, reported that $48.1 \%$ of participants were worried about the safety of the vaccine, $45.2 \%$ expressed disbelief about the COVID-19 vaccine, and $42.6 \%$ were anxious about possible side effects [12]. The fast verification of the safety and effectiveness of the vaccine has been a major concern in many studies from Arab countries as well as in England [10,24]. This represents a big challenge for health authorities as the rapid evolution of the pandemic made the need for a vaccine inevitable. Effective mass education using contextual media preferences in specific countries are required to address the prevailing concerns among the population about the safety of the COVID-19 vaccines. Our study and other studies have reported on the influence of the media (of various types) on vaccine acceptance rates [26,29].

We found that non-Saudi people were less likely to reject the vaccine. This could be due to the fact that non-Saudis would need to be vaccinated to travel to their home countries. Having a previous COVID-19 infection was also associated with high vaccine rejection rates. We had excluded recently infected people because they were ineligible for COVID-19 vaccine in Saudi Arabia. This may indicate a perception among previously infected people of continuing immunity after infection. Educating people about disease and immunity patterns would help address this issue. We found a high income to be associated with higher odds of vaccine rejection. Other studies, however, have reported that lower income was associated with vaccine rejection. Another study from Saudi Arabia reported no association between income and vaccination acceptance [13]. In contrast to our findings, other studies have shown vaccine rejection to be associated with a low monthly income $[24,30]$.

Observational learning is important in adopting something new. This is important in the case of the COVID-19 vaccination campaign as it was rolled out rapidly and people had concerns regarding its safety and efficacy. We found that observing others (friends and family members) was an influencing factor in vaccination decisions. This finding has practical implication as governments can use role models (religious personalities and celebrities) to influence health behaviors of the population for COVID-19 vaccination or for any other disease.

Surprisingly, we did not find government enforcement as significant predictor of vaccine hesitancy. There was a significant increase in the proportion of people accepting vaccination in our data, but when we adjusted for other variables, government mandates became a non-significant variable. This might indicate that some health behaviors, such as COVID-19 vaccination, may not be influenced by enforcement, but rather require an effective informational and educational communication strategy.

We successfully applied SCT to explain COVID-19 vaccine rejection, which is among the few attempts to look at this issue from a social perspective. There are certain limitations that need to considered while interpreting the results of this study. Firstly, the participants were recruited conveniently from PHC centers of one city; therefore, the results may not be generalizable to all of Qassim region or all of Saudi Arabia. Nonetheless, our sample has better population representation than other studies where an online approach was used, which may exclude elderly and illiterate people. Furthermore, our sample is closer to the general population in some of the characteristics such as educational status (up to secondary level $35 \%$ vs. $33 \%$ ) [31], economic participation ( $46 \%$ vs. $49.5 \%$ ) [32], monthly income (SAR 12,500 vs. 14,832 ) [33], and influenza vaccine coverage ( $29 \%$ vs. $37 \%$ ) [34]. Secondly, this was a cross-sectional study that measured the vaccination intention at a single point in time. Rapid evolution of the pandemic, population awareness, and other 
factors might affect this behavior. Thirdly, there could be a possibility of social desirability bias in reporting knowledge and preferences. However, this may have little effects on the validity of our results as we collected anonymized the data through a self-administered questionnaire. Lastly, our sample was powered for COVID-19 vaccine rejection only; therefore, it may not have enough power for all the associations we observed. This is also evident from the wide confidence intervals. Nevertheless, we have explored various individual, social, and environmental factors associated with vaccination behavior.

\section{Conclusions}

Nearly one-third of the participants showed vaccine hesitancy and the most common reason behind vaccine hesitancy was a concern about vaccine safety and effectiveness. This should steer policy makers to develop effective mass health education interventions in order to address disbeliefs and myths related to COVID-19 vaccines among the population by using common channels such as TV/radio and social media. Social and environmental factors also play an important role in modelling vaccination behavior, along with other individual factors. These factors need to be studied and addressed contextually by using health promotion theories such as social cognitive theory.

Author Contributions: Conceptualization, A.A.A. and U.R.; methodology, A.A.A. and U.R.; validation, A.A.A. and U.R.; formal analysis, A.A.A. and U.R.; investigation, A.A.A.; data curation, A.A.A.; writing—original draft preparation, A.A.A.; writing—review and editing, U.R.; visualization, A.A.A. and U.R.; supervision, U.R.; project administration, A.A.A. All authors have read and agreed to the published version of the manuscript.

Funding: This research did not receive any grant from funding agencies in the public, commercial, or not-for-profit sectors.

Institutional Review Board Statement: The study was conducted according to the guidelines of the Declaration of Helsinki and approved by the Qassim Regional Bioethics Committee (1442-1111252, 27 January 2021).

Informed Consent Statement: Written informed consent was obtained from all subjects involved in the study.

Data Availability Statement: Data used in this study can be obtained from corresponding author on request.

Conflicts of Interest: Authors declare that there is no conflict of interest.

\section{References}

1. World Health Organization. General WHO Coronavirus Disease (COVID-19) Dashboard. Available online: https:/ covid19.who. int/table (accessed on 15 February 2021).

2. Chakraborty, C.; Sharma, A.; Sharma, G.; Bhattacharya, M.; Lee, S. SARS-CoV-2 causing pneumonia-associated respiratory disorder (COVID-19): Diagnostic and proposed therapeutic options. Eur. Rev. Med. Pharmacol. Sci. 2020, 24, 4016-4026.

3. Harapan, H.; Wagner, A.L.; Yufika, A.; Winardi, W.; Anwar, S.; Gan, A.K.; Setiawan, A.M.; Rajamoorthy, Y.; Sofyan, H.; Mudatsir, M. Acceptance of a COVID-19 vaccine in Southeast Asia: A cross-sectional study in Indonesia. Front. Public Health 2020, 8, 381. [CrossRef]

4. Wang, J.; Jing, R.; Lai, X.; Zhang, H.; Lyu, Y.; Knoll, M.D.; Fang, H. Acceptance of COVID-19 Vaccination during the COVID-19 Pandemic in China. Vaccines 2020, 8, 482. [CrossRef]

5. Al-Mohaithef, M.; Padhi, B.K. Determinants of COVID-19 vaccine acceptance in Saudi Arabia: A web-based national survey. J. Multidiscip. Healthc. 2020, 13, 1657. [CrossRef] [PubMed]

6. Magadmi, R.M.; Kamel, F.O. Beliefs and barriers associated with COVID-19 vaccination among the general population in Saudi Arabia. BMC Public Health 2021, 21, 1438. [CrossRef]

7. Lazarus, J.V.; Ratzan, S.C.; Palayew, A.; Gostin, L.O.; Larson, H.J.; Rabin, K.; Kimball, S.; El-Mohandes, A. A global survey of potential acceptance of a COVID-19 vaccine. Nat. Med. 2021, 27, 225-228. [CrossRef]

8. Sallam, M. COVID-19 vaccine hesitancy worldwide: A concise systematic review of vaccine acceptance rates. Vaccines 2021, 9, 160. [CrossRef]

9. Kaadan, M.I.; Abdulkarim, J.; Chaar, M.; Zayegh, O.; Keblawi, M.A. Determinants of COVID-19 vaccine acceptance in the Arab world: A cross-sectional study. Glob. Health Res. Policy 2021, 6, 1-7. [CrossRef] [PubMed] 
10. Qunaibi, E.A.; Helmy, M.; Basheti, I.; Sultan, I. A high rate of COVID-19 vaccine hesitancy in a large-scale survey on Arabs. eLife 2021, 10, e68038. [CrossRef] [PubMed]

11. Almaghaslah, D.; Alsayari, A.; Kandasamy, G.; Vasudevan, R. COVID-19 Vaccine Hesitancy among Young Adults in Saudi Arabia: A Cross-Sectional Web-Based Study. Vaccines 2021, 9, 330. [CrossRef]

12. Alobaidi, S. Predictors of Intent to Receive the COVID-19 Vaccination Among the Population in the Kingdom of Saudi Arabia: A Survey Study. J. Multidiscip. Healthc. 2021, 14, 1119. [CrossRef]

13. Mohammed, A.-M.; Padhi, B.K.; Ennaceur, S. Socio-demographics correlate of COVID-19 vaccine hesitancy during the second wave of COVID-19 pandemic: A cross-sectional web-based survey in Saudi Arabia. Front. Public Health 2021, 9, 794.

14. Cheney, M.K.; John, R. Underutilization of influenza vaccine: A test of the health belief model. SAGE Open 2013, 3, 2158244013484732. [CrossRef]

15. De Wit, J.B.; Vet, R.; Schutten, M.; van Steenbergen, J. Social-cognitive determinants of vaccination behavior against hepatitis B: An assessment among men who have sex with men. Prev. Med. 2005, 40, 795-802. [CrossRef] [PubMed]

16. Priest, H.M.; Knowlden, A.P.; Sharma, M. Social cognitive theory predictors of human papillomavirus vaccination intentions of college men at a southeastern university. Community Health Equity Res. Policy 2015, 35, 371-385. [CrossRef]

17. Wong, M.C.S.; Wong, E.L.Y.; Huang, J.; Cheung, A.W.L.; Law, K.; Chong, M.K.C.; Ng, R.W.Y.; Lai, C.K.C.; Boon, S.S.; Lau, J.T.F.; et al. Acceptance of the COVID-19 vaccine based on the health belief model: A population-based survey in Hong Kong. Vaccine 2021, 39, 1148-1156. [CrossRef]

18. Wong, L.P.; Alias, H.; Wong, P.-F.; Lee, H.Y.; AbuBakar, S. The use of the health belief model to assess predictors of intent to receive the COVID-19 vaccine and willingness to pay. Hum. Vaccines Immunother. 2020, 16, 2204-2214. [CrossRef]

19. Shmueli, L. Predicting intention to receive COVID-19 vaccine among the general population using the health belief model and the theory of planned behavior model. BMC Public Health 2021, 21, 804. [CrossRef] [PubMed]

20. Mahmud, I.; Kabir, R.; Rahman, M.A.; Alradie-Mohamed, A.; Vinnakota, D.; Al-Mohaimeed, A. The Health Belief Model Predicts Intention to Receive the COVID-19 Vaccine in Saudi Arabia: Results from a Cross-Sectional Survey. Vaccines 2021, 9, 864. [CrossRef] [PubMed]

21. Zampetakis, L.A.; Melas, C. The health belief model predicts vaccination intentions against COVID-19: A survey experiment approach. Health Well-Being 2021, 13, 469-484. [PubMed]

22. She, R.; Chen, X.; Li, L.; Li, L.; Huang, Z.; Lau, J.T.F. Factors associated with behavioral intention of free and self-paid COVID-19 vaccination based on the social cognitive theory among nurses and doctors in China. Infect. Control Hosp. Epidemiol. 2021, 1-25. [CrossRef] [PubMed]

23. General Authority for Statistics. Sixteenth Services Guide 2017: Qassim Region. Available online: https://www.stats.gov.sa/ sites/default/files/al-qaseem_region_ar.pdf (accessed on 15 January 2020).

24. Bell, S.; Clarke, R.; Mounier-Jack, S.; Walker, J.L.; Paterson, P. Parents' and guardians' views on the acceptability of a future COVID-19 vaccine: A multi-methods study in England. Vaccine 2020, 38, 7789-7798. [CrossRef] [PubMed]

25. Puteh, S.E.W.; Aizuddin, A.N.; Al-Salem, A.A.; Al-shetaily, K.A.; Al-Owayyid, F.H. Attitude, Awareness, and Knowledge of Saudi Citizens towards COVID-19 Vaccination in Qassim Region-Saudi Arabia. Haya Saudi J. Life Sci. 2021, 6, $184-194$.

26. Khaled, S.M.; Petcu, C.; Bader, L.; Amro, I.; Al-Hamadi, A.M.H.; Al Assi, M.; Ali, A.A.M.; Le Trung, K.; Diop, A.; Bellaj, T. Prevalence and Potential Determinants of COVID-19 Vaccine Hesitancy and Resistance in Qatar: Results from a Nationally Representative Survey of Qatari Nationals and Migrants between December 2020 and January 2021. Vaccines 2021, 9, 471. [CrossRef] [PubMed]

27. Lamptey, E.; Serwaa, D.; Appiah, A.B. A nationwide survey of the potential acceptance and determinants of COVID-19 vaccines in Ghana. Clin. Exp. Vaccine Res. 2021, 10, 183-190. [CrossRef]

28. Abebe, H.; Shitu, S.; Mose, A. Understanding of COVID-19 Vaccine Knowledge, Attitude, Acceptance, and Determinates of COVID-19 Vaccine Acceptance Among Adult Population in Ethiopia. Infect. Drug Resist. 2021, 14, 2015. [CrossRef]

29. Piltch-Loeb, R.; Savoia, E.; Goldberg, B.; Hughes, B.; Verhey, T.; Kayyem, J.; Miller-Idriss, C.; Testa, M. Examining the effect of information channel on COVID-19 vaccine acceptance. PLoS ONE 2021, 16, e0251095. [CrossRef]

30. Machida, M.; Nakamura, I.; Kojima, T.; Saito, R.; Nakaya, T.; Hanibuchi, T.; Takamiya, T.; Odagiri, Y.; Fukushima, N.; Kikuchi, H.; et al. Acceptance of a COVID-19 Vaccine in Japan during the COVID-19 Pandemic. Vaccines 2021, 9, 210. [CrossRef]

31. OECD. Education and Tranning: Educational Attainment of 24-64 Years-Olds. Available online: https: / / stats.oecd.org/Index. aspx?datasetcode=EAG_NEAC (accessed on 6 November 2021).

32. General Authority for Statistics. Labour Market Statistics 2021 Q1. Available online: https://www.stats.gov.sa/sites/default/ files/LM_Q1\%202021\%20\%28Press\%20release_EN\%29_0.pdf (accessed on 6 November 2021).

33. General Authority for Statistics. Household Income and Expediture. Available online: https://www.stats.gov.sa/sites/default/ files/nshr_msh_nfq_wdkhl_1sr_2018_nhyy_1-5-2019.pdf (accessed on 6 November 2021).

34. Sagor, K.H.; AlAteeq, M.A. Beliefs, attitudes, and barriers associated with the uptake of the seasonal influenza vaccine among patients visiting primary healthcare clinics. Saudi Med. J. 2018, 39, 690-696. [CrossRef] 